\title{
Association Between Hypoglycemia and the Burden of Comorbidities in Hospitalized Vulnerable Older Diabetic Patients: A Cross-Sectional, Population-Based Study
}

Laure de Decker • Olivier Hanon • Anne-Sophie Boureau • Guillaume Chapelet •

Christelle Dibon $\cdot$ Matthieu Pichelin $\cdot$ Gilles Berrut $\cdot$ Bertrand Cariou

Received: August 22, 2017 / Published online: October 30, 2017

(C) The Author(s) 2017. This article is an open access publication

\begin{abstract}
Introduction: From a patient-centered perspective, the assessment of risk factors of hypoglycemia is of critical importance for the management of type 2 diabetes (T2D). However, the association between the occurrence of hypoglycemia and high burden of comorbidities has been poorly studied in vulnerable older
\end{abstract}

Enhanced content To view enhanced content for this article go to http://www.medengine.com/Redeem/ 24CCF060418BED36.

L. de Decker · G. Chapelet

Laboratory of clinical and experimental therapeutics of infections, EA 3628, Nantes University, 44000

Nantes, France

L. de Decker · Anne-SophieBoureau - G. Chapelet .

C. Dibon - G. Berrut

Department of Geriatrics, Nantes University

Hospital, 44000 Nantes, France

O. Hanon

Department of Geriatrics, Broca Hospital, Public

Hospital of Paris, 75013 Paris, France

\section{O. Hanon}

Laboratory of Alzheimer disease : genetic and vascular markers, neuropsychology, psychosocial interventions and technologies, EA 4468, Paris Descartes University, Sorbonne Paris Cité, 75006 Paris, France

\section{Pichelin · B. Cariou $(\bowtie)$}

Department of Endocrinology, l'institut du thorax, Nantes University Hospital, 44000 Nantes, France e-mail: bertrand.cariou@univ-nantes.fr patients. Here, we aimed to determine whether a high burden of comorbidities is associated with hypoglycemia in very old patients with T2D.

Methods: A total of 1552 elderly (age $\geq 80$ years old) patients with T2D were recruited in a nationwide cross-sectional study performed in French geriatric care units. Hypoglycemia was defined as a confirmed blood glucose value level $\leq 70 \mathrm{mg} / \mathrm{dL}$. Comorbidities were assessed using the Charlson Comorbidity Index (CCI).

Results: Amongst the 1552 recruited patients (mean age 86.4 years), 415 (26.7\%) had documented hypoglycemia. Compared to patients in whom hypoglycemia was not reported, they have a lower body weight $(p=0.004)$, a reduced eGFR $(p<0.001), \quad$ a greater level of dependency $(p<0.001)$ as well as history of dementia $(p=0.006)$ and cardiovascular disease $(p<0.001)$, and a higher CCI (4.7 vs 3.8, $p<0.001)$. Patients with hypoglycemia had a higher frequency of daily self-monitoring blood glucose (SMBG) $(p<0.001)$ and insulin use $(p<0.001)$, with reduced sulfonylurea use $(p<0.001)$. In multivariate logistic regression analysis, insulin therapy (OR 3.32, $p<0.001$ ), daily SMBG (OR 1.79, $p=0.02$ ), CCI (OR 1.24, $p=0.01$ ), and age (OR $0.96, p=0.03)$ were independently associated with the risk of hypoglycemia.

Conclusion: In addition to insulin therapy, a high burden of comorbidities was independently associated with hypoglycemia in older vulnerable patients with T2D. 
Keywords: Charlson comorbidity index; Comorbidities; Diabetes; Hypoglycemia; Older patients

\section{INTRODUCTION}

Type 2 diabetes (T2D) is a common disease in older patients [1]. Hypoglycemia is one of the major complications of diabetes, especially among the vulnerable older patients [2, 3]. Hypoglycemia has been suggested to be a vulnerability marker of older patients since it is associated with all-cause mortality, stroke, heart failure, myocardial infarction, and cognitive functional disorders [3-5]. However, hypoglycemia in this population is generally less diagnosed than in younger adults [6]. Numerous factors have been associated with the risk of hypoglycemia in older patients, such as low social status, long duration of diabetes, malnutrition, polypharmacy, eating disorders, $\mathrm{HbA}_{1 \mathrm{C}}$ level, cognitive and functional disorders, kidney failure, falls, stroke, cancer, and cardiovascular diseases (CVD) [7, 8].

Older adults' health status is heterogeneous because of cumulative effects of acute and chronic diseases contributing to high burden of comorbidities [9].

Different scales have been developed to score the burden of comorbidities, defined as pathologies that influence the occurrence or the evolution of an index pathology, biological factors, and social conditions [10]. The Charlson Comorbidity Index (CCI) is a standardized and validated method for scoring burden of comorbidities and predicting mortality by weighting chronic conditions [11]. Two studies in the general population have highlighted the deleterious role of a high level of burden of comorbidities in patients hospitalized for accidents or in those under insulin therapy $[12,13]$.

Currently, the association between the occurrence of hypoglycemia and high burden of comorbidities in the very old population ( $>80$ years) has been poorly studied.

Here, we assessed the correlation between a high level of burden of comorbidities measured by the CCI and the occurrence of hypoglycemia in a large number of patients with $\mathrm{T} 2 \mathrm{D}$ over 80 years old, hospitalized in geriatric care units.

\section{METHODS}

\section{Participants}

A cross-sectional survey conducted by the French Society of Geriatrics and Gerontology (SFGG) included subjects with T2D on June 21, 2012. This original study included patients aged over 80 years and hospitalized in French geriatric care units (geriatric acute-care service, post-acute, acute care and rehabilitation, and nursing home). Patients with T2D were excluded if they had no antidiabetic treatment (insulin or/and glinide or/and metformin or/and sulfonylureas or/and dipeptidyl peptidase-4 inhibitors or/and alpha-glucosidase inhibitors, or/and glucagon-like peptide-1 receptor agonists).

\section{Clinical Assessment}

A standardized questionnaire was sent by e-mail to 1500 practitioners working in geriatric care units. Physician-reported responses were received from 324 practitioners. Occurrence of hypoglycemia (response was yes or no) in elderly patients with T2D was recorded by the physicians during their hospitalization period in geriatric units. Confirmed hypoglycemia was defined as a blood glucose level $\leq 70 \mathrm{mg} / \mathrm{dL}$, assessed either with venous sampling or capillary self-monitoring blood glucose (SMBG) [14]. The timing and the number of hypoglycemic episodes per patient were not recorded in the questionnaire.

Data collection included factors thought to be associated with hypoglycemia, as suggested by previous studies [7, 8, 15-19]. For each patient, the following information were collected: gender, age, weight (kg), living in a nursing home, the number of concomitant medications, systolic and diastolic blood pressure $(\mathrm{mmHg})$, depression, dementia, cardiovascular diseases (atrial fibrillation, heart failure, peripheral arterial disease, or coronary heart 
disease), stroke, cancer, history of falls, antihypertensive treatment [angiotensin converting enzyme (ACE) inhibitors and angiotensin receptor blockers (ARBs), beta-blockers, diuretics, calcium channel blockers (CCBs)]; as well as antidiabetic treatment: insulin therapy, oral antidiabetic drugs (OADs) (metformin, sulfonylureas, glinides), and SMBG frequency. As biological markers, fasting plasma glucose (FPG), $\mathrm{HbA}_{1 \mathrm{C}}$, and the estimated glomerular filtration rate (eGFR, $\mathrm{mL} / \mathrm{min}$ ) calculated using the Cockcroft-Gault formula were collected.

The burden of comorbidities was evaluated using the CCI [11]. Comorbidities were defined as acute or chronic co-existing diseases referring to an index pathology at the time of the study [11]. The CCI is widely used in studies of older patients and demonstrates strong inter-judge reliability and good reproducibility [11]. The CCI score is the sum of the weightings for all the patient's conditions. We obtained the total score by adding the points for all 19 relevant pathological statuses. The total score is a continuous variable ranging from 0 to 30 .

\section{Standard Protocol Approval, Registration, and Patient Consent}

All procedures followed were in accordance with the ethical standards of the responsible committee on human experimentation and with the Helsinki Declaration of 1964, as revised in 2013. This study was approved by the ethical committee of Nantes and performed in accordance with French law. The entire study protocol was approved by the local Ethical Committee of Nantes (France), and the study was in compliance with the STROBE statement guidelines. Waiving of consent was authorized for this study in accordance with French law owing to the non-interventional nature of the study.

\section{Statistics}

The patients' baseline characteristics were summarized using means and standard deviations, or frequencies and percentages, as appropriate. Normality of the data distribution was checked using the skewness-kurtosis test.
Because the number of observations was greater than 40 for each group, no transformations were applied to the variables of interest. Patients were separated into two groups based on the presence or absence of hypoglycemia. Between-group comparisons were performed using an independent sample $t$ test or Chi square test, as appropriate. Univariate and multivariate logistic regressions were performed to identify the association between hypoglycemia (dependent variable) and a CCI score (independent variable) and adjusted for the patients' baseline characteristics (factors with a $p$ value less than 0.20 in univariate analysis and confounding factors); $p$ values less than 0.05 were considered to indicate statistical significance. All statistics were performed using SPSS (version17.0; SPSS, Inc., Chicago, IL).

\section{RESULTS}

A total of 1552 patients (69.7\% female) were included in this survey, with a mean age of $86.4 \pm 4.4$ years. We identified 415 patients $(26.7 \%)$ with documented hypoglycemia. The clinical characteristics of the included subjects are summarized in Table 1 . The hypoglycemic group consisted mainly of women $(n=293$; $70.6 \%)$, mean age $86.3 \pm 4.5$ years old. They had a lower body weight $(p=0.004)$, a greater level of dependency $(p<0.001)$, a reduced eGFR $(p<0.001)$, and a higher frequency of dementia $(p=0.006)$ and CVD $(p<0.001)$ compared with those who did not experience a hypoglycemic event. In addition, a higher proportion of older patients with T2D and hypoglycemia reported a daily SMBG compared to those who did not experience a hypoglycemic event $(p<0.001)$. Regarding the antidiabetic treatment, the results showed a higher prevalence of insulin use $(p<0.001)$ and a lower prevalence of oral antidiabetic drug prescription (sulfonylureas, $p<0.001$; glinides, $p=0.002$; metformin, $p<0.001)$ in patients reporting hypoglycemic episodes. Finally, older subjects who experienced hypoglycemia had a higher CCI compared to those without hypoglycemia (4.7 vs $3.8, p<0.001)$. All the results of the descriptive analyses are presented in Table 1. 
Table 1 Comparison of baseline characteristics of patients with and without hypoglycemia $(n=1552)$

\begin{tabular}{|c|c|c|c|}
\hline \multirow[t]{2}{*}{ Characteristics } & \multicolumn{2}{|l|}{ Hypoglycemia } & \multirow{2}{*}{$p$ value $^{\mathrm{a}}$} \\
\hline & Yes $(n=415)$ & No $(n=1137)$ & \\
\hline Age (years), mean $\pm S D$ & $86.1 \pm 4.5$ & $86.5 \pm 4.4$ & 0.190 \\
\hline Female, $n(\%)$ & $293(70.6)$ & $788(69.4)$ & 0.639 \\
\hline Weight $(\mathrm{kg})$, mean $\pm \mathrm{SD}$ & $65.8 \pm 14.7$ & $69.5 \pm 24.5$ & 0.004 \\
\hline Systolic blood pressure $(\mathrm{mmHg})$, mean $\pm \mathrm{SD}$ & $130.7 \pm 18$ & $129.8 \pm 16.8$ & 0.357 \\
\hline Diastolic blood pressure $(\mathrm{mmHg})$, mean $\pm \mathrm{SD}$ & $70.3 \pm 11.8$ & $69.5 \pm 11.9$ & 0.301 \\
\hline Renal function, eGFR $(\mathrm{mL} / \mathrm{min})$, mean $\pm \mathrm{SD}^{\mathrm{b}}$ & $46.1(20.8)$ & $51.9(26.1)$ & $<0.001$ \\
\hline Living in nursing home, $n(\%)$ & $284(68.7)$ & $776(68.2)$ & 0.873 \\
\hline $\mathrm{HbA}_{1 \mathrm{C}}(\%)$, mean $\pm \mathrm{SD}^{\mathrm{c}}$ & $7.73 \pm 1.3$ & $7.54 \pm 3.3$ & 0.308 \\
\hline Fasting plasma glucose $(\mathrm{mg} / \mathrm{dl})$, mean $\pm \mathrm{SD}$ & $159 \pm 14$ & $148 \pm 12$ & 0.146 \\
\hline Daily SMBG, ${ }^{\mathrm{d}} n(\%)$ & $351(84.6)$ & $771(67.8)$ & $<0.001$ \\
\hline Insulin, $n(\%)$ & $360(86.7)$ & $717(63.1)$ & $<0.001$ \\
\hline Glinides, $n(\%)$ & $31(7.5)$ & $151(13.3)$ & $<0.002$ \\
\hline Metformin, $n(\%)$ & $48(11.6)$ & $256(22.5)$ & $<0.001$ \\
\hline Sulfonylureas, $n$ (\%) & $22(5.3)$ & $160(14.1)$ & $<0.001$ \\
\hline Beta-blockers, $n$ (\%) & $121(29.5)$ & $318(28.2)$ & 0.619 \\
\hline ACE inhibitors and ARBs, $n$ (\%) & $185(45.5)$ & $523(46.6)$ & 0.688 \\
\hline Diuretics, $n(\%)$ & $165(40.3)$ & $428(38)$ & 0.407 \\
\hline Calcium channel blockers, $n(\%)$ & $118(28.9)$ & $299(26.6)$ & 0.364 \\
\hline Number of co-medications, mean $\pm S D$ & $8.52 \pm 3.3$ & $8.62 \pm 3.2$ & 0.610 \\
\hline Cardiovascular diseases ${ }^{\mathrm{e}}, n(\%)$ & $278(68.6)$ & $608(54.3)$ & $<0.001$ \\
\hline Stroke, $n(\%)$ & $133(32.7)$ & $311(27.7)$ & 0.057 \\
\hline Cancer, $n(\%)$ & $55(13.4)$ & $147(13)$ & 0.840 \\
\hline Depression, $n(\%)$ & $160(38.9)$ & $384(34)$ & 0.072 \\
\hline Falls, $n(\%)$ & $139(33.7)$ & $338(29.9)$ & 0.159 \\
\hline Dementia, $n(\%)$ & $276(67)$ & $672(59.3)$ & 0.006 \\
\hline Charlson comorbidity index, score, mean \pm SD & $4.7(2.3)$ & $3.8(2.1)$ & $<0.001$ \\
\hline
\end{tabular}

${ }^{a}$ Based on independent samples $t$ test or Chi square test as appropriate, with significance, $p<0.05$; significant $p$ values are indicated in bold

b $e$ GFR estimated glomerular filtration rate

${ }^{c} H b A_{1 C}$ glycated hemoglobin

d $S M B G$ self-monitoring blood glucose

e Atrial fibrillation or heart failure or peripheral arterial disease 
Table 2 Univariate and multivariate logistic regression models showing the association between hypoglycemia (dependent variable) and Charlson comorbidity index (independent variable) adjusted for clinical characteristics $(n=1552)$

\begin{tabular}{|c|c|c|c|c|c|c|}
\hline & \multicolumn{3}{|c|}{ Univariate model } & \multicolumn{3}{|c|}{ Multivariate model } \\
\hline & $\overline{\mathrm{OR}}$ & $95 \% \mathrm{CI}$ & $p$ value $^{\mathrm{a}}$ & $\overline{\text { OR }}$ & $95 \% \mathrm{CI}$ & $p$ value $^{a}$ \\
\hline Age, years & 0.98 & {$[0.96-1.01]$} & 0.190 & 0.96 & [0.92-0.99] & 0.026 \\
\hline Female & 1.06 & {$[0.83-1.36]$} & 0.639 & 1.01 & {$[0.71-1.44]$} & 0.953 \\
\hline Weight & 0.98 & {$[0.98-0.99]$} & $<0.001$ & 0.99 & {$[0.98-1.00]$} & 0.177 \\
\hline Systolic blood pressure & 1.00 & [0.99-1.01] & 0.357 & - & - & - \\
\hline Diastolic blood pressure & 1.00 & {$[0.99-1.01]$} & 0.301 & - & - & - \\
\hline Renal function, $\mathrm{eGFR}^{\mathrm{b}}$ & 0.99 & {$[0.98-0.99]$} & $<0.001$ & 0.99 & {$[0.98-1.00]$} & 0.068 \\
\hline Living in nursing home & 1.02 & {$[0.80-1.30]$} & 0.873 & - & - & - \\
\hline $\mathrm{HbA}_{1 \mathrm{C}}{ }^{\mathrm{c}}$ & 1.02 & {$[0.98-1.06]$} & 0.332 & 0.99 & {$[0.93-1.06]$} & 0.870 \\
\hline Fasting plasma glucose & 1.06 & {$[0.98-1.16]$} & 0.153 & 1.07 & {$[0.96-1.20]$} & 0.212 \\
\hline Daily SMBG & 3.17 & {$[2.32-4.33]$} & $<0.001$ & 1.79 & {$[1.09-2.96]$} & 0.021 \\
\hline Insulin & 3.83 & {$[2.81-5.22]$} & $<0.001$ & 3.32 & {$[1.88-5.86]$} & $<0.001$ \\
\hline Glinides & 0.53 & {$[0.35-0.79]$} & 0.002 & 0.68 & {$[0.38-1.24]$} & 0.211 \\
\hline Metformin & 0.45 & {$[0.32-0.63]$} & $<0.001$ & 0.77 & [0.46-1.29] & 0.327 \\
\hline Sulfonylureas & 0.34 & {$[0.22-0.54]$} & $<0.001$ & 1.18 & {$[0.60-2.32]$} & 0.622 \\
\hline Beta-blockers & 1.06 & {$[0.83-1.37]$} & 0.619 & 0.95 & {$[0.67-1.34]$} & 0.758 \\
\hline ACE inhibitors and ARBs & 0.95 & {$[0.76-1.2]$} & 0.688 & 0.88 & {$[0.64-1.21]$} & 0.446 \\
\hline Diuretics, $n$ (\%) & 1.10 & {$[0.87-1.39]$} & 0.407 & - & - & - \\
\hline Calcium channel blockers, $n$ (\%) & 1.25 & {$[0.87-1.45)$} & 0.357 & - & - & - \\
\hline Number of co-medications & 0.98 & {$[0.93-1.04]$} & 0.562 & 0.98 & {$[0.93-1.03]$} & 0.390 \\
\hline Cardiovascular diseases $^{\mathrm{d}}$ & 1.84 & {$[1.45-2.30]$} & $<0.001$ & 1.35 & {$[0.94-1.94]$} & 0.106 \\
\hline Stroke & 1.27 & {$[0.99-1.62]$} & 0.056 & 0.91 & {$[0.64-1.29]$} & 0.600 \\
\hline Cancer & 1.03 & {$[0.74-1.44]$} & 0.840 & 0.74 & {$[0.43-1.26]$} & 0.271 \\
\hline Depression & 1.24 & {$[0.98-1.56]$} & 0.073 & 1.17 & {$[0.85-1.61]$} & 0.326 \\
\hline Falls & 1.19 & {$[0.93-1.51]$} & 0.159 & 1.26 & {$[0.91-1.74]$} & 0.169 \\
\hline Dementia & 1.39 & {$[1.10-1.77]$} & 0.006 & 1.15 & {$[0.82-1.61]$} & 0.403 \\
\hline Charlson comorbidity index & 1.18 & {$[1.12-1.24]$} & $<0.001$ & 1.24 & [1.02-1.23] & 0.014 \\
\hline
\end{tabular}

${ }^{a}$ Based on independent samples $t$ test or Chi square test as appropriate, with significance, $p<0.05$; significant $p$ values are indicated in bold

${ }^{\mathrm{C}} \mathrm{CrCl}$ creatinine clearance

c $H b A_{1 C}$ glycated hemoglobin

${ }^{\mathrm{d}}$ Atrial fibrillation or heart failure or peripheral arterial disease 
In the unadjusted model, CCI was significantly associated with hypoglycemia [odds ratio (OR), 1.18; 95\% confidence interval (CI, 1.12-1.24); $p<0.001]$ (Table 2).

In adjusted multivariate regression analysis, hypoglycemia was independently associated with insulin therapy (OR 3.32; 95\% CI 1.88-5.86; $p<0.001$ ), daily SMBG (OR 1.79; $95 \%$ CI $1.09-2.96 ; p=0.021$ ), CCI (OR 1.24; $95 \%$ CI 1.02-1.23; $p=0.014$ ), and age (OR 0.96; 95\% CI 0.92-0.99; $p=0.026$ ) (Table 2).

\section{DISCUSSION}

The occurrence of hypoglycemic events is very common in older patients with diabetes. Since the results from the large randomized trails such as ACCORD and VADT have clearly highlighted a positive association between symptomatic and severe hypoglycemia and the risk of death, the prevention of hypoglycemia becomes a priority in the management of $\mathrm{T} 2$, especially in the elderly [21, 22]. Here, we specifically assessed the factors associated to the risk of hypoglycemia in vulnerable very old ( $>80$ years) patients hospitalized in geriatric care units.

One of the most interesting results of our real-life study is that a high burden of comorbidities, measured by CCI, is an independent predictor of hypoglycemia in this population. Furthermore, age, frequency of daily SMBG, and insulin therapy were also significantly associated with hypoglycemia. In contrast, our findings indicate that $\mathrm{HbA}_{1 \mathrm{C}}$, kidney function, dementia, or OADs use were not independently associated with hypoglycemia in this elderly population.

To the best of our knowledge, no previous studies have examined the association between the burden of comorbidities and hypoglycemia in patients older than 80 years. It has been reported that the burden of comorbidities, measured by CCI, is associated with hypoglycemia in an unadjusted model especially among patients hospitalized for accidents and among patients aged over 50 years with insulin-treated type 2 diabetes [12, 13]. Interestingly, a recent prospective study conducted in 363 patients with diabetes older than 65 years has demonstrated that frailty indices, including Frailty Trait Score (FTS) and Frailty Index (FI), were independently associated with the risk of death [23].

In our study, we found no association between specific comorbidities and hypoglycemia. This result was contradictory with most previous studies focused on specific comorbidities such as depression, dementia, CVD, stroke, cancer, history of falls, hypertension, liver cirrhosis, or renal diseases [15-19]. Two methodological reasons might explain this discrepancy. First, in these studies, specific comorbidities differ in each adjusted model. Second, few studies have incorporated the CCI calculated from specific comorbidities in adjusted statistic models. The CCI score is a better marker of an individual's morbidity status, especially in older patients, because it reflects the burden comorbidity, rather than a single aspect of one comorbidity. Altogether, this suggests that the CCI score, which includes the effects of all comorbidities, appears to be a consistent measure for assessing the vulnerability of older patients with T2D and thus identifying those with the higher risk of hypoglycemia.

One surprising result is that the youngest patients in our cohort were more likely to experience a hypoglycemic event. These results are surprising considering previous studies [1-3]. Two methodological reasons can explain this observation. First, patients studied were 80 years of age or older and mostly lived in nursing home unlike patients in other studies $[2,3]$. Second, the $95 \%$ CI for the OR is close to 1 and therefore this statistical difference appears to be not clinically relevant in this very old population.

Daily SMBG was positively associated with hypoglycemia. These results are consistent with previous studies [24, 25]. Indeed, the close glucose monitoring with continuous glucose monitoring (CGM) was associated with a higher number of hypoglycemic events [24, 25]. In addition, we cannot exclude that undiagnosed hypoglycemia also occurs in patients that were unable to perform daily SMBG, as suggested by some studies using CGM [26]. For instance, in the study by Munshi et al., $69 \%$ of the patients 
(mean age 75 years) had at least one nocturnal hypoglycemic episode. Importantly, 93\% of hypoglycemic episodes were unrecognized by SMBG measurements performed four times a day or by symptoms [26].

Importantly, insulin therapy was the most powerful independent predicting factor associated with hypoglycemia. This result is consistent with previous studies $[3,17,27]$ and insulin therapy is the second biggest cause of emergency hospitalization for adverse drug events in older Americans [28]. This reinforces the clinical need for education of the caregivers to prevent the occurrence of hypoglycemia in older subjects with diabetes treated with insulin, as well as the development of new insulin analogues with a lower risk of hypoglycemia.

In contrast to the current findings in international literature, we found no independent association between level of $\mathrm{HbA}_{1 \mathrm{C}}$, OADs (mainly sulfonylureas), and hypoglycemia. These discrepant results may be explained by methodological differences between the studies $[7,8]$. First, the population included in all the previous studies was younger than ours. Second, there are fewer confounding factors-notably comorbidities. Third, for OADs, the majority of studies did not reveal if these treatments were in mono-therapy or combination therapy and at what doses, which can affect the results $[3,7]$.

One can also hypothesize that OADs were predominantly used in patients with lesser comorbidities, and thus at lower risk of hypoglycemia, than those under insulin therapy. Indeed, we found in our study that elderly subjects on sulfonylureas had a significantly lower CCI score than those who were not [3.3 $( \pm 2.1)$ vs $4.2( \pm 2.2) ; p<0.001]$. Thus, the paradoxical lower risk of hypoglycemia in patients under sulfonylureas is certainly driven by a lower level of burden of comorbidities in our elderly population.

Despite the inclusion of a large sample $(>1500)$ of very old patients with diabetes, a rare situation in the literature, the present study had some limitations. A reporting bias may be included in the dataset. Indeed, the accuracy and completeness of our data were entirely reliant upon physicians' declarations. However, the questionnaire was designed to limit variability in readers' interpretations by asking only factual data. Regarding the hypoglycemia, the exact timing of hypoglycemia during the hospitalization, including the relationship with some potential changes in antidiabetic treatments, has not been recorded. Although we were able to control several characteristics that may have modified the association, residual potential confounders might still be present. For instance, we were not able to control factors related to low social status, duration of diabetes, nutritional status, or eating disorders.

\section{CONCLUSION}

This study highlights the fact that a high burden of comorbidities was associated with increased hypoglycemia risk in elderly vulnerable patients with T2D. Thus, older patients with numerous comorbid conditions may require greater attention from practitioners. Efforts to extend our knowledge of the risk factors associated with hypoglycemia should be continued in order to improve the care of elderly diabetic patients from a patient-centered perspective.

\section{ACKNOWLEDGEMENTS}

The article processing charges were funded by the CHU de Nantes, France (CIC Endocrino-Nutrition). This study was supported by the French Society of Geriatrics and Gerontology (SFGG). All authors had full access to all of the data in this study and take complete responsibility for the integrity of the data and accuracy of the data analysis. All named authors meet the International Committee of Medical Journal Editors (ICMJE) criteria for authorship for this manuscript, take responsibility for the integrity of the work as a whole, and have given final approval for the version to be published. We are grateful to the participants for their cooperation, particularly each geriatric care unit.

Disclosures. Bertrand Cariou has received research funding from Pfizer and 
Sanofi-Regeneron, received honoraria from Amgen, AstraZeneca, Pierre Fabre, Janssen, Eli-Lilly, MSD Merck \& Co., Novartis, Novo-Nordisk, Sanofi, and Takeda. Laure de Decker, Olivier Hanon, Anne-Sophie Boureau, Guillaume Chapelet, Christelle Dibon, Matthieu Pichelin, and Gilles Berrut have nothing to disclose.

Compliance with Ethics Guidelines. All procedures followed were in accordance with the ethical standards of the responsible committee on human experimentation and with the Helsinki Declaration of 1964, as revised in 2013. This study was approved by the ethical committee of Nantes and performed in accordance with French law.

Data Availability. The datasets generated and/or analyzed during the current study are available from the corresponding author on reasonable request. This article is distributed under the terms of the Creative Commons Attribution-NonCommercial 4.0 International License (http://creativecommons.org/licenses/ by-nc/4.0/), which permits any noncommercial use, distribution, and reproduction in any medium, provided you give appropriate credit to the original author(s) and the source, provide a link to the Creative Commons license, and indicate if changes were made.

Open Access. This article is distributed under the terms of the Creative Commons Attribution-NonCommercial 4.0 International License (http://creativecommons.org/licenses/ by-nc/4.0/), which permits any noncommercial use, distribution, and reproduction in any medium, provided you give appropriate credit to the original author(s) and the source, provide a link to the Creative Commons license, and indicate if changes were made.

\section{REFERENCES}

1. Guariguata L, Whiting DR, Hambleton I, Beagley J, Linnenkamp U, Shaw JE. Global estimates of diabetes prevalence in adults for 2013 and projections for 2035 for the IDF Diabetes Atlas. Diabetes Res Clin Pract. 2013;103(2):137-49.

2. Frier BM. Hypoglycaemia in diabetes mellitus: epidemiology and clinical implications. Nat Rev Endocrinol. 2014;10(12):711-22.

3. Abdelhafiz AH, Rodríguez-Mañas L, Morley JE, Sinclair AJ. Hypoglycemia in older people-a less well recognized risk factor for frailty. Aging Dis. 2015;6(2):156-67.

4. Garg R, Hurwitz S, Turchin A, Trivedi A. Hypoglycemia, with or without insulin therapy, is associated with increased mortality among hospitalized patients. Diabetes Care. 2013;36(5):1107-10. doi:10.2337/dc12-1296.

5. McCoy RG, Van Houten HK, Ziegenfuss JY, Shah ND, Wermers RA, Smith SA. Increased mortality of patients with diabetes reporting severe hypoglycemia. Diabetes Care. 2012;35(9):1897-901. doi:10.2337/dc11-2054.

6. Matyka K, Evans M, Lomas J, Cranston I, Macdonald I, Amiel SA. Altered hierarchy of protective responses against severe hypoglycemia in normal aging in healthy men. Diabetes Care. 1997;20(2):135-41.

7. Yanai H, Adachi H, Katsuyama H, Moriyama S, Hamasaki H, Sako A. Causative anti-diabetic drugs and the underlying clinical factors for hypoglycemia in patients with diabetes. World J Diabetes. $2015 ; 6(1): 30-6$.

8. Davis KL, Wei W, Meyers JL, Kilpatrick BS, Pandya N. Association between different hemoglobin A1c levels and clinical outcomes among elderly nursing home residents with type 2 diabetes mellitus. J Am Med Dir Assoc. 2014;15(10):757-62.

9. Feinstein AR. Pre-therapeutic classification of co-morbidity in chronic disease. J Chronic Dis. 1970;23:455-68.

10. Valderas JM, Starfield B, Sibbald B, Salisbury C, Roland M. Defining comorbidity: implications for understanding health and health services. Ann Fam Med. 2009;7:357-63.

11. Charlson ME, Pompei P, Ales KL, MacKenzie CR. A new method of classifying prognostic comorbidity in longitudinal studies: development and validation. J Chronic Dis. 1987;40:373-83.

12. Signorovitch JE, Macaulay D, Diener $M$, et al. Hypoglycaemia and accident risk in people with type 2 diabetes mellitus treated with non-insulin antidiabetes drugs. Diabetes Obes Metab. 2013;15:335-41. 
13. Kostev K, Dippel FW, Rathmann W. Predictors of hypoglycaemia in insulin-treated type 2 diabetes patients in primary care: a retrospective database analysis. Prim Care Diabetes. 2014;8(2):127-31.

14. American Diabetes Association Workgroup on Hypoglycemia. Defining and reporting hypoglycemia in diabetes: a report from the American Diabetes Association Workgroup on Hypoglycemia. Diabetes Care. 2005;28(5):1245-9.

15. Abbatecola AM, Bo M, Barbagallo M, et al. Severe hypoglycemia is associated with antidiabetic oral treatment compared with insulin analogs in nursing home patients with type 2 diabetes and dementia: results from the DIMORA study. J Am Med Dir Assoc. 2015;16(4):349.e7-12.

16. Boucai L, Southern WN, Zonszein J. Hypoglycemia-associated mortality is not drug-associated but linked to comorbidities. Am J Med. 2011;124(11):1028-35.

17. Kagansky N, Levy S, Rimon E, et al. Hypoglycemia as a predictor of mortality in hospitalized elderly patients. Arch Intern Med. 2003;163(15):1825-9.

18. Seufert J, Brath H, Pscherer S, Borck A, Bramlage P, Siegmund T. Composite efficacy parameters and predictors of hypoglycaemia in basal-plus insulin therapy-a combined analysis of 713 type 2 diabetic patients. Diabetes Obes Metab. 2014;16(3):248-54.

19. Holstein A, Patzer OM, Machalke K, Holstein JD, Stumvoll M, Kovacs P. Substantial increase in incidence of severe hypoglycemia between 1997-2000 and 2007-2010: a German longitudinal population-based study. Diabetes Care. 2012;35(5):972-5.

20. Hsu PF, Sung SH, Cheng HM, et al. Association of clinical symptomatic hypoglycemia with cardiovascular events and total mortality in type 2 diabetes: a nationwide population-based study. Diabetes Care. 2013;36(4):894-900.
21. Bonds DE, Miller ME, Bergenstal RM, et al. The association between symptomatic, severe hypoglycaemia and mortality in type 2 diabetes: retrospective epidemiological analysis of the ACCORD study. BMJ. 2010;340:b4909.

22. Tkác I. Effect of intensive glycemic control on cardiovascular outcomes and all-cause mortality in type 2 diabetes: overview and metaanalysis of five trials. Diabetes Res Clin Pract. 2009;86(Suppl 1):S57-62.

23. Castro-Rodríguez M, Carnicero JA, Garcia-Garcia FJ, et al. Frailty as a major factor in the increased risk of death and disability in older people with diabetes. J Am Med Dir Assoc. 2016;17(10):949-55.

24. Pazos-Couselo M, García-López JM, González-Rodríguez $\mathrm{M}$, et al. High incidence of hypoglycemia in stable insulin-treated type 2 diabetes mellitus: continuous glucose monitoring vs. self-monitored blood glucose. Observational prospective study. Can J Diabetes. 2015;39(5):428-33.

25. Gómez AM, Umpierrez GE, Muñoz OM, et al. Continuous glucose monitoring versus capillary point-of-care testing for inpatient glycemic control in type 2 diabetes patients hospitalized in the general ward and treated with a basal bolus insulin regimen. J Diabetes Sci Technol. 2015;10(2):325-9.

26. Munshi MN, Segal AR, Suhl E, et al. Frequent hypoglycemia among elderly patients with poor glycemic control. Arch Intern Med. 2011;171(4):362-4.

27. Cariou B, Fontaine P, Eschwege E, et al. Frequency and predictors of confirmed hypoglycaemia in type 1 and insulin-treated type 2 diabetes mellitus patients in a real-life setting: results from the DIALOG study. Diabetes Metab. 2015;41(2):116-25.

28. Budnitz DS, Lovegrove MC, Shehab N, Richards CL. Emergency hospitalizations for adverse drug events in older Americans. N Engl J Med. 2011;365(21):2002-12. 